\title{
Effects of interplanetary shock inclinations on nightside auroral power intensity
}

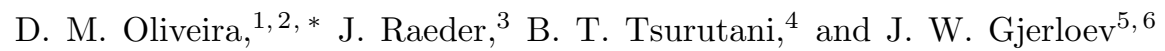 \\ ${ }^{1}$ NASA Goddard Space Flight Center, Greenbelt MD, USA \\ ${ }^{2}$ Goddard Planetary Heliophysics Institute, University of Maryland Baltimore County, Baltimore MD, USA \\ ${ }^{3}$ EOS Space Science Center and Department of Physics, \\ University of New Hampshire, Durham NH, USA \\ ${ }^{4}$ Jet Propulsion Laboratory, California Institute of Technology, Pasadena CA, USA \\ ${ }^{5}$ Johns Hopkins University Applied Physics Laboratory, Laurel MD, USA \\ ${ }^{6}$ Birkeland Centre of Excellence, University of Bergen, Norway
}

\begin{abstract}
We derive fast forward interplanetary (IP) shock speeds and impact angles to study the geoeffectiveness of 461 IP shocks that occurred from January 1995 to December 2013 using ACE and Wind spacecraft data. The geomagnetic activity is inferred from the SuperMAG project data. SuperMAG is a large chain which employs more than 300 ground stations to compute enhanced versions of the traditional geomagnetic indices. The SuperMAG auroral electroject SME index, an enhanced version of the traditional AE index, is used as an auroral power (AP) indicator. AP intensity jumps triggered by shock impacts are correlated with both shock speed and impact angle. It is found that high AP intensity events typically occur when high speed IP shocks impact the Earth's magnetosphere with the shock normal almost parallel to the Sun-Earth line. This result suggests that symmetric and strong magnetospheric compression leads to favorable conditions for intense auroral power release, as shown previously by simulations and observations. Some potential mechanisms will be discussed.
\end{abstract}

Keywords: Space physics, ionosphere-magnetosphere interaction, plasma physics.

\section{INTRODUCTION}

Interplanetary (IP) shocks result from the interaction of solar disturbances with the ambient solar wind [40]. As they propagate throughout the heliosphere, IP shocks eventually interact with different bodies in the solar system, such as planets, moons, and even asteroid5 s. In the eventual cases in which IP shocks strike Earth, they interact with the Earth's magnetosphere, causing disturbances that can be detected in the near-Earth space environment, the whole magnetosphere and even the ionosphere. The first dramatic magnetospheric effect associated with IP shock impacts is the $\mathrm{SSC} / \mathrm{SI}^{+}$(storm sudden commencement/sudden impulse), resulting from the sudden magnetospheric/magnetotail compression and the Earthward motion of the Chapman- Ferraro current. Other effects following shock-related $\mathrm{SSC} / \mathrm{SI}^{+}$events may also occur: geomagnetic storms [13], radiation belt perturbations [16, 57], and GICs (ground-induced currents). GICs may pose risks to electric power transmission systems leading to power grid disruptions and serious economic losses [4, 10, 17, 46]. GICs are also associated with corrosion of pipelines and their control systems [14].IP shocks are well known to sometimes trigger substorms as well [45].

IP shocks are well known to sometimes trigger substorms as well $3,6,6,9,18-20,23-25,45,54,56]$. In the early days before shock detection in interplanetary space, $\mathrm{SSCs} / \mathrm{SI}^{+} \mathrm{s}$ were used to imply the impingement of an IP shock or tangential discontinuity (TD) onto the magnetosphere as an attempt to explain geomagnetic activity

*Electronic address: denny@umbc.edu following $\mathrm{SSC} / \mathrm{SI}^{+}$events. As shown later by 19], who statistically examined $\mathrm{SSC} / \mathrm{SI}^{+}$events, intense au- roral activity always occurred when $\mathrm{SSC} / \mathrm{SI}^{+}$amplitudes were greater than $40 \mathrm{nT}$. [48] later showed that most of $\mathrm{SSCs} / \mathrm{SI}^{+} \mathrm{S}$ were caused by IP shocks rather than TDs. Precursor IMF $B_{z}$ events $\sim 1.5 \mathrm{hr}$ prior to shock arrival have bee used to identify when shocks would be geoeffective and when they would not be $6,6,921,51,54-56]$

Another important factor of IP shock geoeffectiveness is the IP shock impact angle, which is the angle between the shock normal vector and the Sun-Earth line. IP shocks with shock normals almost aligned with the SunEarth line are typically driven by CMEs (coronal mass ejections) [40] whereas inclined shocks tend to be driven by CIRs (corotating interaction regions) more frequently [38, 47]. Several studies addressing geomagnetic activity following inclined IP shocks have been done in the past. For example, a longer than usual SSC rise time caused by the impact of an IP shock with the Earth's magnetosphere was observed by Wind spacecraft [49]. These authors argued that this effect should be related to the high inclination of the shock normal in the equatorial plane. Due to its high impact angle, the IP shock took a longer time to sweep over the magnetosphere, compressing it gradually leading to a slow magnetospheric response. The authors suggested that simulation studies with the impact of inclined IP shocks on the Earth's magnetosphere should be carried out. This suggestion was taken by other authors [15] who simulated the impact of two similar IP shocks, one inclined and the other frontal, on the Earth's magnetosphere. They found that both systems evolved to very similar final quasi-steady states, although in the inclined case the system took more time to evolve in relation to the head-on case. Very similar 
results were found with MHD numerical simulations by other studies as well [42, 44, 52]. A statistical study with more than 300 fast forward IP shocks was performed [53] to study the same effects, i.e., the effect of IP shock inclinations on the SSC rise time followed by IP shock impacts. They found that IP shocks impacting the Earth's magnetosphere with shock normals almost aligned with the Sun-Earth line caused short SSC rise times when compared to inclined IP shock events. Their highest correlation occurred when the shocks were strong and almost head-on. These simulation and statistical results confirmed the observation and suggestion made previously by observations [49] and numerical simulations as well [15, 42, 44, 52].

Impact of IP shocks on the Earth's magnetosphere were investigated in other simulation studies [41, 43] with an MHD code especially developed to study effects generated on the magnetosheath [41]. In this case, the shock impact was frontal and the magnetospheric response was symmetric. Later, using the same MHD code, one of the previous authors a simulated case of the interaction of a similar IP shock, but with an inclined shock normal, with the Earth's magnetosphere. He found that the inclined shock took more time to travel through the magnetosheath in comparison to his previous studies. This author argued that inclined shocks with large downstream $v_{y}$ component may generate asymmetries on both dawn and dusk sides leading to non-symmetric magnetospheric compressions. Such compressions can lead to different SSC amplitudes depending upon the side of impact. He then suggested that such effects should be detectable by magnetometers on the ground.

More recently, the impact of IP shocks with different shock normal inclinations on the Earth's magnetosphere were simulated [31, 33]. Using the OpenGGCM MHD code [39], these authors simulated three different cases, namely, two inclined and one frontal. The second inclined shock was twice as stronger as the other inclined shock, and the frontal shock had the same strength as the first inclined shock. The shock normals of the two inclined shocks lay in the meridian plane. The same authors reported that the head-on shock was more geoeffective than the inclined shocks, even more geoeffective than the strong inclined shock. For example, the frontal shock triggered substorm signatures and high nightside auroral energy dissipations not seen in the inclined cases. They suggested that the frontal shock, whose shock normal was aligned with the Sun-Earth line, compressed the magnetotail symmetrically on both north and south sides. Such condition created an ideal scenario for the energy stored in the magnetotail to be leaked away and trigger auroral substorms at Earth [33, 34]. These results suggested the same authors to look for these effects in satellite and geomagnetic data.

The sequence of this simulation work was conducted by the same authors [35, 36], who performed a statistical study of IP shock properties at 1 AU using a shock list with events from January 1995 to December 2013. They found that the yearly number of IP shocks is well correlated with solar activity, confirming previous observations [8, 30]. Although shocks occur more frequently during solar maxima due to the higher occurrence of CMEs, CIRs tend to drive most shocks in solar minima. Due to the fact that CMEs tend to drive frontal shocks and CIRs tend to drive more inclined shocks, more frontal events tend to occur during solar maxima. They also reported that the majority of shocks found in the heliosphere at 1 AU are weak shocks, with Mach numbers less than 3. In the same statistical study, they studied the effects of IP shock impact angles on substorm strength indicated by an enhanced version of the AL index. They found that almost frontal shocks were generally more geoeffective than inclined shocks. Their strongest correlation was found in the case when shocks with high speed, or strong shocks, impacted the Earth almost frontally.

The goal of this paper is to study geomagnetic activity triggered by IP shocks in correlation to IP shock speeds and impact angles. Here the geomagnetic activity is represented by auroral power (AP) intensity as inferred from an enhanced version of the auroral electroject index AE. Correlations are obtained from shock speed and impact angles using the same IP shock list published by a shock statistical study [35], the most extensive fast forward shock study done to date. In the following, in section 2 , we present the data. In section 3 , we report our results, which are summarize and briefly discussed in section 4.

\section{DATA ANALYSES}

Our data analysis is based on a list of 461 fast forward IP shocks from Wind and ACE data from January 1995 up to December 2013 published by a recent statistical study 35]. The shock normal orientations were obtained from different shock normal determination methods, such as the well known magnetic and velocity coplanarity methods [5], and the formulas that mix plasma and IMF data [1, 2]. All shocks were required to satisfy the Rankine-Hugoniot conditions [32, 50].

We use the SuperMAG geomagnetic station data to identify auroral power associated with shock impingement. SuperMAG [1] is an international collaboration with a chain of more than 300 ground stations used to compute the SME, SMU, and SML indices 27, 28], the enhanced versions of $\mathrm{AE}, \mathrm{AU}$, and $\mathrm{AL}$ 7], respectively. The SuperMAG indices are very similar to the traditional IAGA indices: their main difference is the fact that the former are computed based on data of a larger number of ground stations in comparison to the latter. The SuperMAG data were obtained from the websites http://supermag.jhuapl.edu/ and http://supermag.uib.no/. Technical issues related to SuperMAG data analysis and assimilation were detailed in a paper entirely devoted to this subject [12].

The SME index is used as a proxy for aurora power 
(AP) determinations. This choice was based on a relation found by other authors [28]. These authors calibrated the SME index with both Polar UVI instantaneous images and DMSP instantaneous maps to obtain possible correlations between SME and AP. Due to time resolutions issues, the most relevant correlation found by them was between SME and AP as determined by Polar UVI. The linear relationship found in this previous work and used here is:

$$
A P=0.048 \times S M E+0.241 \times(S M E)^{1 / 2},
$$

where AP is represented in $\mathrm{GW}$, and the square root portion comes from the monoenergetic auroral contribution. In equation (1), AP was integrated over the northern hemisphere polar cap between 1800-0600 magnetic local time and $60^{\circ}$ and $80^{\circ}$ magnetic latitude. More specifically, expression (1) indicates the nightside AP intensity as calculated from the SuperMAG SME index. Later, the SME index was confirmed to be the best choice to predict AP intensity instead of SMU and SML [29].

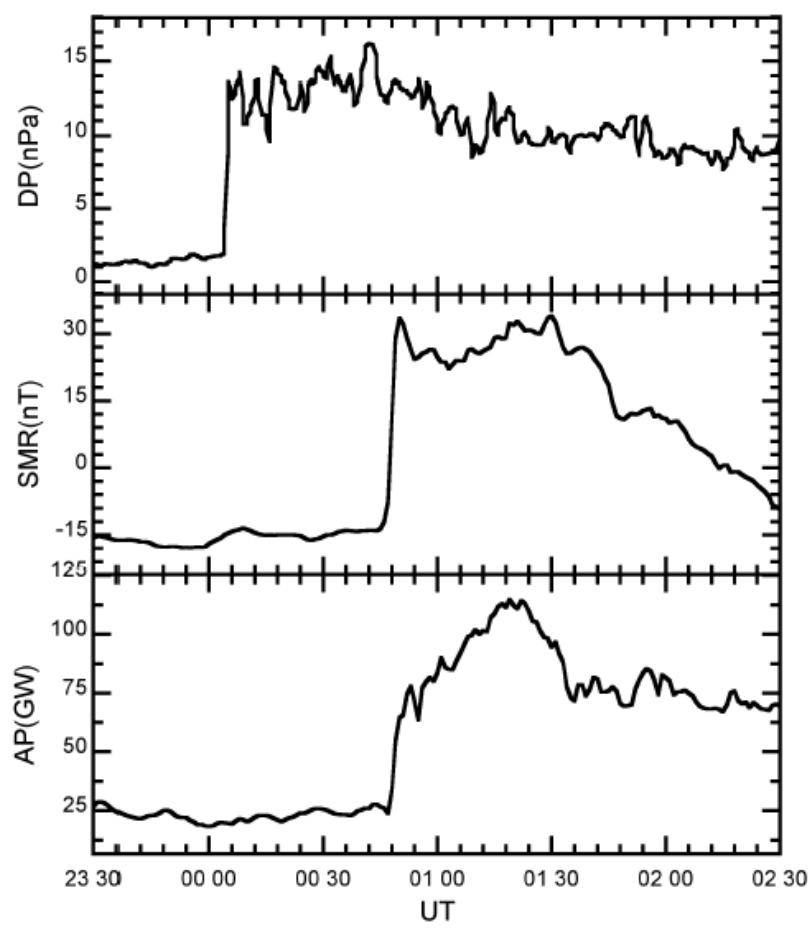

Figura 1: ACE observation of an interplanetary shock on 18 April 2001 at 0005 UT and its consequent geomagnetic activity. Top panel shows increase in dynamic pressure $\rho v^{2}$ (DP, in $\mathrm{nPa}$ ) observed by ACE. Nearly 50 minutes later, as shown in the middle panel, the Earth's magnetopause is struck by the IP shock, as indicated by SuperMAG ground stations with the sharp increase in the SMR index, in nT. Finally, the bottom panel shows the increase in $\mathrm{AP}(\mathrm{GW})$ approximately 35 minutes after shock impact.
The methodology used to record geomagnetic activity followed by IP shock impacts is shown by Figure 1. This figure represents an IP shock from the shock list compiled by a previous statistical study 35]. At 0005 UT on 18 April 2001, ACE observed a sharp jump in the dynamic pressure $\mathrm{DP}=\rho v^{2}$ upstream of the Earth. After approximately 50 minutes, the IP shock impinged on the Earth's magnetopause, and a sharp jump in SMR, the SuperMAG measurement associated with the ring current [26], was recorded by SuperMAG ground stations. Then, in the next 40 minutes, the SuperMAG ground stations registered a peak in the SME index, which was used to plot AP in the last panel of Figure 1 according to equation (1). For all events in our statical analysis, the maximum measurements in AP followed by IP shock impacts were recorded in the time lag of 2 hours after shock impacts. If there are more than one AP peak in this time interval, the first one is chosen as the maximum associated with the IP shock. More details can be found in a previous work 35 ].

\section{RESULTS}

Figure 2 represents the statistical results obtained from our 461 IP shock events. Figure 2(a) shows the distribution of $\theta_{x_{n}}$, the shock impact angle between the shock normal and the GSE Sun-Earth line. Angles close to $180^{\circ}$ indicate that the shock normal vector is almost parallel to the Sun-Earth line. Most shocks had $\theta_{x_{n}}$, indicating that they typically range from moderately inclined to almost frontal shocks. Figure 2(b) represents the distribution of shock speed vs in our shock list. The average shock speed is about $500 \mathrm{~km} / \mathrm{s}$ in the Earth's frame of reference, and most shocks have vs below the average. This result indicates that the IP shocks observed in the heliosphere at 1 AU are predominantly weak IP shocks with Mach numbers between 1 and 3. More details about the statistical results of the IP shocks in our database can be found in a previous paper [35].

Correlations of variations in auroral power, $\Delta \mathrm{AP}$, in GW, with the shock speed $v_{s}$, in $\mathrm{km} / \mathrm{s}$, is shown in Figure 3. For this parameter selection, the impact angle $\theta_{x_{n}}$ is held in constant intervals while the shock speed is allowed to vary. The data are binned in three different categories: Figure $3(\mathrm{a}), 120^{\circ} \leq \theta_{x_{n}} \leq 140^{\circ}$, highly inclined shocks; 2(b), $140^{\circ}<\theta_{x_{n}} \leq 160^{\circ}$, moderately inclined shocks; and $2(\mathrm{c}), 160^{\circ}<\theta_{x_{n}} \leq 180^{\circ}$, almost frontal shocks. Here we consider events with low auroral activity when $\Delta \mathrm{AP}<20 \mathrm{GW}$, and events with high auroral activity when $\triangle \mathrm{AP}$ i, $80 \mathrm{GW}$. Events with moderate auroral activity are between these two limits. Figure 3(a) shows that most highly inclined shock events with low auroral activity are associated with weak, low speed $\left(v_{s}<450\right.$ 

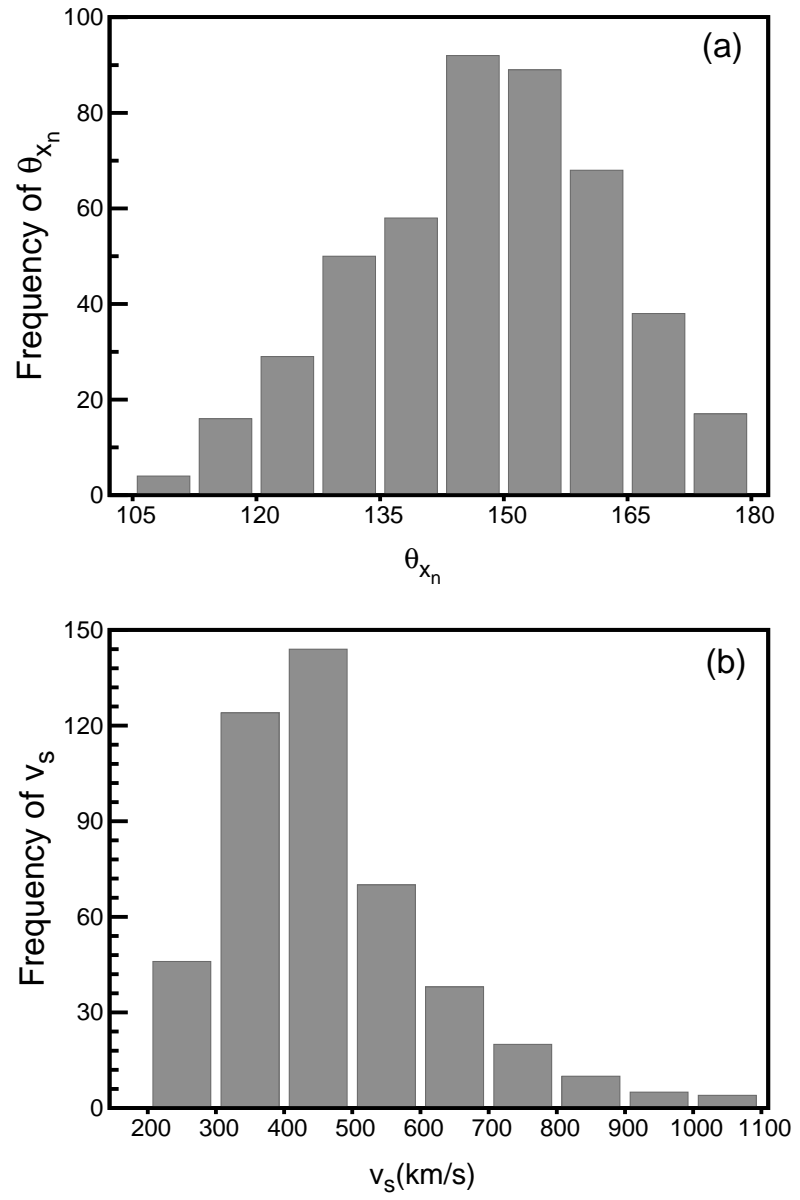

Figura 2: Statistical results of fast forward IP shocks observed by Wind and ACE from January 1995 to December 2013. Figure 1(a) shows the shock impact angle distribution, and Figure 1(b) represents shock speed $(\mathrm{km} / \mathrm{s})$ distribution.

$\mathrm{km} / \mathrm{s})$ shocks. Strong, high speed $\left(v_{s}>550 \mathrm{~km} / \mathrm{s}\right)$ shocks are related to events with moderate auroral activity, with only one event that has low auroral activity being caused by a strong shock. Events with moderate auroral activity are associated with all shock strength categories with approximately the same likelihood. There are no events with high auroral activity triggered by highly inclined shocks in our database. The correlation coefficient in this case is $\mathrm{R}=0.45$ and the average of AP is 25.98 GW.

The intermediate category of shock strength has the largest number of events, as seen in Figure 3(b). In this case, all events with low auroral activity are triggered by weak or low speed shocks. Most events with moderate activity are associated with weak or moderate shocks. All events with high auroral activity are triggered by high speed shocks. The correlation coefficient is $\mathrm{R}=0.55$ and $\overline{\mathrm{AP}}=41.37 \mathrm{GW}$. Figure 3(c) shows that all weak auroral activity events (only three cases) are related to weak shocks. Events with moderate auroral activity are mostly associated with weak or moderate shocks, but some
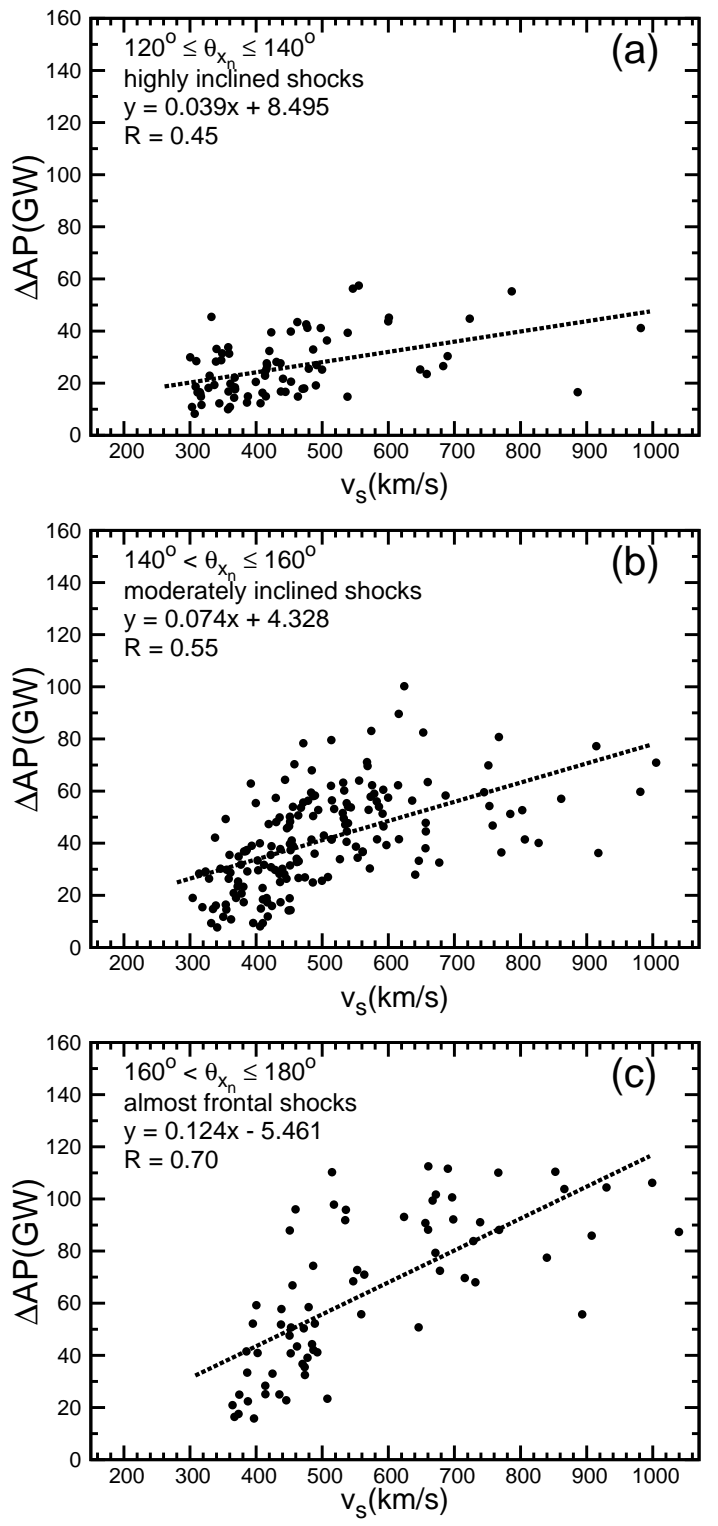

Figura 3: Auroral power amplitude as a function of shock speed binned in three different shock impact angle categories: (a), $120^{\circ} \leq \theta_{x_{n}} \leq 140^{\circ}$; (b) $140^{\circ}<\theta_{x_{n}} \leq 160^{\circ}$; and (c), $160^{\circ}$ $<\theta_{x_{n}} \leq 180^{\circ}$.

are related to strong shocks. All events with intense auroral activity are triggered by either moderate or strong shocks. The correlation coefficient $\mathrm{R}=0.70$ and the average $\overline{\mathrm{AP}}=64.09 \mathrm{GW}$ are the highest in this category. These results are summarized in Table 1.

A comparison among the three cases described above shows that, on aver- age, $\Delta \mathrm{AP}$ increases with shock speed when the impact angle is close to 180o. The correlation coefficient between the shock speed and $\Delta \mathrm{AP}$ also increases for almost frontal shocks. 
The opposite analysis is made in Figure 4, i.e., where the shock impact angles are allowed to vary keeping the shock speed binned in constant intervals. The three categories are: Figure $4(\mathrm{a}), 300 \leq v_{s} \leq 450 \mathrm{~km} / \mathrm{s}$, weak shocks; $450<v_{s} \leq 550 \mathrm{~km} / \mathrm{s}$, moderate shocks; and $v_{s}$ i. $550 \mathrm{~km} / \mathrm{s}$, strong shocks. Figure 4(a) shows that weak shocks are associated with events with either weak or moderate auroral activity, and are not related to events with intense auroral activity. There are only a few weak highly inclined shocks, and most of them cause events with moderate auroral activity. Only a few strong highly inclined shocks cause events with low auroral activity. The correlation coefficient for highly inclined shocks, $\mathrm{R}=0.39$, and the average of $26.55 \mathrm{GW}$, are the lowest in this case. In the category of moderate shocks, the correlation is stronger, with $\mathrm{R}=0.48$, and the average is higher, with $\overline{\mathrm{AP}}=46.56 \mathrm{GW}$. There are only a few events with low auroral activity, and most of them are triggered by highly inclined shocks and just a few by inclined shocks. Moderate almost frontal shocks, seen in Figure 4(b), triggered either moderate or strong auroral activity events. Typically, events with moderate auroral activity are triggered by moderate, strong, and weak shocks. There are only a few events with high auroral activity, and all of them are triggered by moderate almost frontal shocks. Finally, correlations for strong shock are represented by Figure 4(c). Generally, strong shocks do not cause events with low auroral activity, with an exception of only one event caused by a highly inclined shock. Events with moderate AP activity are typically caused by inclined shocks, but they can also be triggered by highly inclined or almost frontal shocks. Events with intense auroral activity 235 are caused mostly by almost frontal shocks, but a few events are caused by inclined shocks. The correlation coefficient and AP average for strong shocks are the highest in this category, $\mathrm{R}=0.79$ and $\overline{\mathrm{AP}}=62.88 \mathrm{GW}$. Table 1 summarizes the results obtained for correlations with shocks in all categories.

The analysis of the three panels in Figure 4 leads to a similar conclusion obtained in the analysis of Figure 3: strong, high speed shocks are generally much more geoeffective than weak slow speed shocks, and their geoeffectiveness increases if the IP shock impacts more frontally on the Earth's magnetosphere. These general results were predicted previously in global MHD simulations [33] and confirmed experimentally with spacecraft and geomagnetic data observations [35].

\section{SUMMARY AND CONCLUSIONS}

We have studied 461 fast forward interplanetary (IP) shocks using Wind and ACE satellite data from January 1995 to December 2013. We correlated IP shock impact angles with geomagnetic activity (auroral power intensity) triggered by IP shock impacts. The primary result
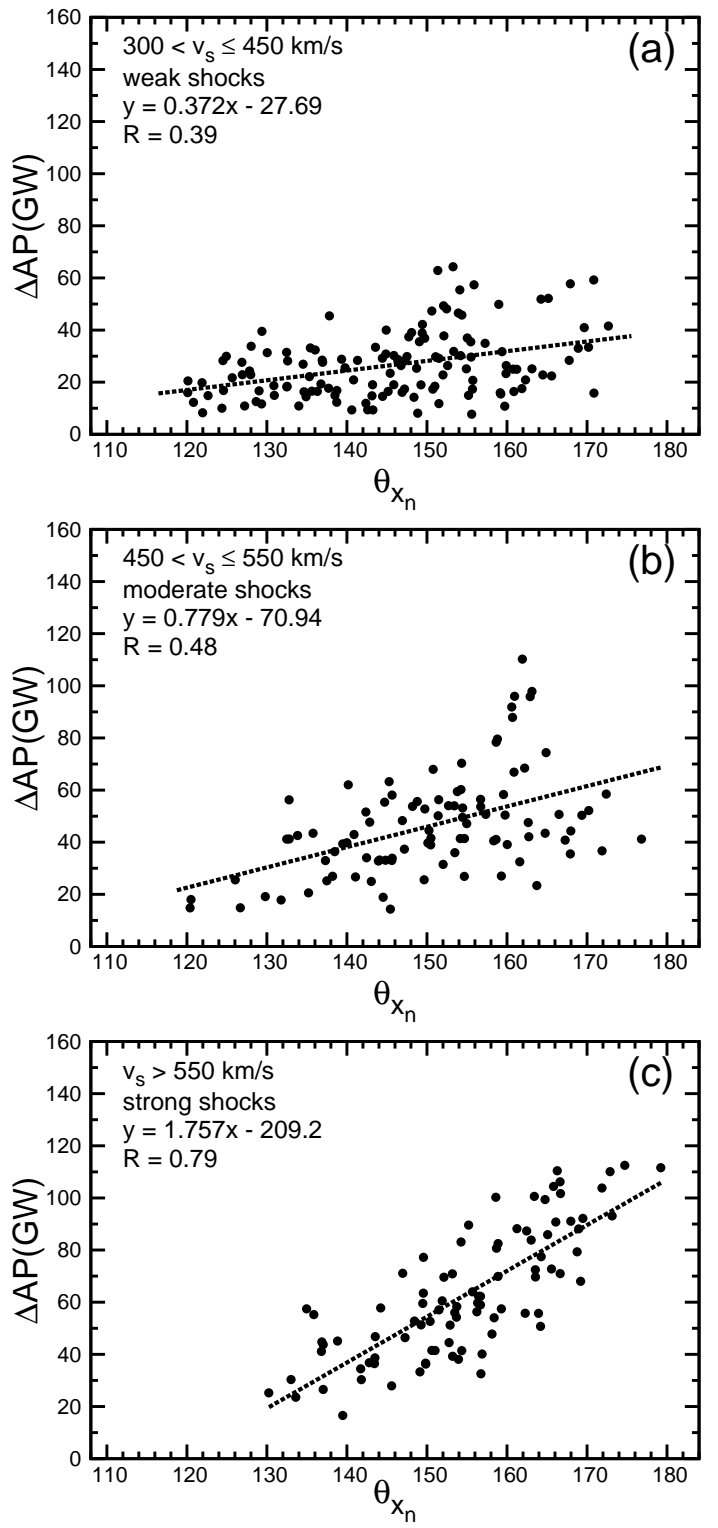

Figura 4: Auroral power amplitude as a function of shock impact angle binned in three different shock speed categories: (a), $300 \leq v_{s} \leq 450 \mathrm{~km} / \mathrm{s}$; (b) $450<v_{s} \leq 550 \mathrm{~km} / \mathrm{s}$; and (c), $v_{s}>550 \mathrm{~km} / \mathrm{s}$.

Fixed impact angle $\theta_{x_{n}}$, changed shock speed $v_{s}$

category highly inclined moderately inclined almost frontal

\begin{tabular}{|c|c|c|c|}
\hline $\mathrm{R}$ & 0.45 & 0.55 & 0.70 \\
\hline$\overline{\mathrm{AP}}$ & 25.98 & 41.37 & 64.09 \\
\hline \multicolumn{4}{|c|}{ Fixed shock speed $v_{s}$, changed impact angle $\theta_{x_{n}}$} \\
\hline ategory & weak & moderate & strong \\
\hline $\mathrm{R}$ & 0.39 & 0.48 & 0.79 \\
\hline$\overline{\mathrm{AP}}$ & 26.55 & 46.56 & 62.88 \\
\hline
\end{tabular}

Tabela I: Summary of the results obtained for the shock speed, shock impact angle, and $\triangle \mathrm{AP}$ correlation analyses. 
obtained here was that high speed shocks with shock normal aligned along the Sun-Earth line (head-on shocks) cause the greatest auroral power release. The correlation coefficient for the cross correlation analysis in this case was 0.79 , the highest of any performed in this study. This result confirms previous numerical simulation results [33], in which frontal shocks led to stronger geomagnetic activity in comparison to the cases of inclined IP shocks. Observational results were reported in a subsequent work [35], whose authors performed a statistical analysis correlating substorm strength and IP shock impact angles. In the case of fast (strong) shocks, events with the strongest geomagnetic activity occurred in the cases in which the shocks impacted the magnetopause almost frontally.

To explain the above results, it should be first noted that shock compression of the magnetosphere is most effective when the inclination angle is frontal. Both the magnetosphere and magnetotail will be compressed the most for this orientation. Greater tail lobe fields will require stronger cross tail currents to maintain them. Magnetosphere/magnetotail compression will lead to more flattened tail closed field lines. Shock-triggeringsubstorm mechanisms were previously discussed by several other authors 3, 51, 54, 55]. Both current disruption [22, 23, 37] and magnetic reconnection [19, 23 25] are viable under these above conditions.

The present results indicate the role of shock speed and inclination angle in geoeffectiveness of magnetospheric energy release (auroral power). Thus this is another factor besides magnetospheric priming that must be taken into account in assessing auroral power release.

\section{Acknowledgments}

This work was supported by grant AGS-1143895 from the National Science Foundation and grant FA-9550120264 from the Air Force Office of Sponsored Research. We thank the Wind and ACE teams for the solar wind data and CDAWeb interface for data availability. We thank Dr. C. W. Smith, the ACE team, and Dr. J. C. Kasper for their list compilations. For the ground magnetometer data we gratefully acknowledge: Intermagnet; USGS, Jeffrey J. Love; CARISMA, PI Ian Mann; CANMOS; The S-RAMP Database, PI K. Yumoto and Dr. K. Shiokawa; The SPIDR database; AARI, PI Oleg Troshichev; The MACCS program, PI M. Engebretson, Geomagnetism Unit of the Geological Survey of Canada; GIMA; MEASURE, UCLA IGPP and Florida Institute of Technology; SAMBA, PI Eftyhia Zesta; 210 Chain, PI K. Yumoto; SAMNET, PI Farideh Honary; The institutes who maintain the IMAGE magnetometer array, PI Eija Tanskanen; PENGUIN; AUTUMN, PI Martin Conners; DTU Space, PI Dr. Jürgen Matzka; South Pole and McMurdo Magnetometer, PI's Louis J. Lanzarotti and Alan T. Weatherwax; ICESTAR; RAPIDMAG; PENGUIn; British Artarctic Survey; McMac, PI Dr. Peter Chi; BGS, PI Dr. Susan Macmillan; Pushkov Institute of Terrestrial Magnetism, Ionosphere and Radio Wave Propagation (IZMIRAN); GFZ, PI Dr. Jürgen Matzka; MFGI, PI B. Heilig; IGFPAS, PI J. Reda; University of L'Aquila, PI M. Vellante; SuperMAG, PI Jesper W. Gjerloev. D.M.O. thanks the SuperMAG PI J. W. Gjerloev for the straightforward SuperMAG website for its convenience of data visualization and download.
[1] Abraham-Shrauner, B. (1972), Determination of magnetohydronamic shock normals, J. Geophys. Res., 77(4), 736-739, doi:10.1029/JA077i004p00736.

[2] Abraham-Shrauner, B., and S. H. Yun (1976), Interplanetary shocks seen by Ames Plasma Probe on Pioneer 6 and 7, J. Geophys. Res., 81(13), doi:10.1029/ JA081i013p02097.

[3] Akasofu, S.-I., and J. Chao (1980), Interplanetary shock waves and magnetospheric substorms, Planet. Space Sci., 28(4), 381-385, doi:10.1016/0032-0633(80)90042-2.

[4] Bolduc, L. (2002), GIC observations and studies in the Hydro-Québec power system, J. Atmos. Sol. Terr. Phys., 64 (16), 1793-1802, doi:10.1016/S1364-6826(02)00128-1.

[5] Colburn, D. S., and C. P. Sonett (1966), Discontinuities in the solar wind, Space Sci. Rev., 439(A11), 506, doi: 10.1007/BF00240575.

[6] Craven, J. D., L. A. Frank, C. T. Russell, E. E. Smith, and R. P. Lepping (1986), Global auroral responses to magnetospheric compressions by shocks in the solar wind: Two case studies, in Solar Wind-Magnetosphere Coupling, edited by Y. Kamide and J. A. Slavin, pp. 367-380, Terra Scientific, Tokyo, Japan.

[7] Davis, T. N., and M. Sugiura (1966), Auroral electrojet activity index $\mathrm{AE}$ and its universal time varia- tions, J. Geophys. Res., 71(3), 785-801, doi:10.1029/ JZ071i003p00785.

[8] Echer, E., W. D. Gonzalez, L. E. A. Vieira, A. D. L. F. L. Guarnieri, A. Prestes, A. L. C. Gonzalez, and N. J. Schuch (2003), Interplanetary shock parameters during solar activity maximum (2000) and minimum (1995-1996), Braz. Jour. Phys., 33(1), 2301, doi: 10.1590/S0103-97332003000100010.

[9] Echer, E., B. Tsurutani, F. Guarnieri, and J. Kozyra (2011), Interplanetary fast forward shocks and their geomagnetic effects: CAWSES events, J. Atmos. Sol. Terr. Phys., 73(11-12), 1330-1338, doi:10.1016/j.jastp.2010. 09.020 .

[10] Erinmez, I. A., J. G. Kappenman, and W. A. Radasky (2002), Management of the geomagnetically induced current risks on the national grid company's electric power transmission system, J. Atmos. Sol. Terr. Phys., 63(56), 743-756, doi:10.1016/S1364-6826(02)00036-6.

[11] Gjerloev, J. W. (2009), A global ground-based magnetometer initiative, Eos Trans. AGU, 90(27), 230-231, doi: $10.1029 / 2009 \mathrm{EO} 270002$.

[12] Gjerloev, J. W. (2012), The SuperMAG data processing technique, J. Geophys. Res., 117(A09213), 1-19, doi:10. 1029/2012JA017683. 
[13] Gonzalez, W. D., B. T. Tsurutani, and A. L. Clúa de Gonzalez (1999), Interplanetary origin of geomagnetic storms, Space Sci. Rev., 88(3-4), 529-562, doi: 10.1023/A:1005160129098.

[14] Gummow, R. A., and P. Eng (2002), GIC effects on pipeline corrosion and corrosion control systems, J. Atmos. Sol. Terr. Phys., 64(16), 1755-1764, doi:10.1016/ S1364-6826(02)00125-6.

[15] Guo, X.-C., Y.-Q. Hu, and C. Wang (2005), Earth's magnetosphere impinged by interplanetary shocks of different orientations, Chinese Phys. Lett., 22(12), 3221-3224, doi: 10.1088/0256-307X/22/12/067.

[16] Halford, A. J., S. L. McGregor, K. R. Murphy, R. M. Millan, M. K. Hudson, L. A. Woodger, C. A. Cattel, A. W. Breneman, I. R. Mann, W. S. Kurth, G. B. Hospodarsky, M. Gkioulidou, and J. F. Fennell (2015), BARREL observations of an ICME-shock impact with the magnetosphere and the resultant radiation belt electron loss, J. Geophys. Res., 120(4), 2557-2570, doi: 10.1002/2014JA020873.

[17] Kappenman, J. (2010), Geomagnetic storms and their impacts on the US power grid, Tech. rep., Metatech Corp., Goleta, California.

[18] Kawasaki, K., S.-I. Akasofu, F. Yasuhara, and C.-I. Meng (1971), Storm sudden commencements and polar magnetic substorms, J. Geophys. Res., 76, 6781-6789, doi: 10.1029/JA076i028p06781.

[19] Kokubun, S., R. L. McPherron, and C. T. Russell (1977), Triggering of substorms by solar wind discontinuities, J. Geophys. Res., 82(1), 74-86, doi:10.1029/ JA082i001p00074.

[20] Liou, K., P. T. Newell, C.-I. Meng, C.-C. Wu, and R. P. Lepping (2003), Investigation of external triggering of substorms with Polar ultraviolet imager observations, J. Geophys. Res., 108(A10), doi:10.1029/2003JA009984.

[21] Liu, J.-J., H.-Q. Hu, D.-S. Han, Z.-Y. Xing, Z.-J. Hu, D.-H. Huang, and H.-G. Yang (2013), Response of nightside aurora to interplanetary shock from ground optical observation, Chinese J. Geophys, 56(5), 598-611, doi: $10.1002 / \mathrm{cjg} 2.20056$.

[22] Lui, A. T. Y., R. E. Lopez, S. M. Krimigis, R. W. McEntire, L. J. Zanetti, and T. A. Potemra (1988), A case study of magnetotail current sheet disruption and diversion, Geophys. Res. Lett., 15(7), 721-724, doi: 10.1029/GL015i007p00721.

[23] Lui, A. T. Y., A. Mankofsky, C.-L. Chang, K. Papadopoulos, and C. S. Wu (1990), A current disruption mechanism in the neutral sheet: A possible trigger for substorm expansions, J. Geophys. Res., 17(6), 745-748, doi:10.1029/GL017i006p00745.

[24] Lyons, L. R. (1995), A new theory for magnetospheric substorms, J. Geophys. Res., 100(A10), 19,069-19,081, doi:10.1029/95JA01344.

[25] Lyons, L. R. (1996), Substorms: Fundamental observational features, distinction from other disturbances, and external triggering, J. Geophys. Res., 101 (A6), 13,01113,025, doi:10.1029/95JA01987.

[26] Newell, P. T., and G. W. Gjerloev (2012), SuperMAGbased partial ring current indices, J. Geophys. Res., 117(A05215), 1-15, doi:10.1029/2012JA017586.

[27] Newell, P. T., and J. W. Gjerloev (2011), Evaluation of SuperMAG auroral electrojet indices as indicators of substorms and auroral power, J. Geophys. Res., 116(A12), doi:10.1029/2011JA016779.
[28] Newell, P. T., and J. W. Gjerloev (2011), Substorm and magnetosphere characteristic scales inferred from the SuperMAG auroral electrojet indices, J. Geophys. Res., 116(A12211), 1-12, doi:10.1029/2011JA016936.

[29] Newell, P. T., and J. W. Gjerloev (2014), Local geomagnetic indices and the prediction of auroral power, J. Geophys. Res., 119(12), 9790-9803, doi:10.1002/ 2014JA020524.

[30] Oh, S. Y., Y. Yi, and Y. H. Kim (2007), Solar cycle variation of the interplanetary forward shock drivers observed at 1 AU, Solar Phys., 245(2), 391-410, doi: 10.1007/s11207-007-9042-2.

[31] Oliveira, D., and J. Raeder (2013), Role of symmetry in the geo-effectiveness of interplanetary shocks, in $S M 41 B$ 2232, AGU Fall Meeting, San Francisco, CA.

[32] Oliveira, D. M. (2015), A study of interplanetary shock geoeffectiveness controlled by impact angles using simulations and observations, Ph.D. thesis, University of New Hampshire.

[33] Oliveira, D. M., and J. Raeder (2014), Impact angle control of interplanetary shock geoeffectiveness, J. Geophys. Res., 119 (10), 8188-8201, doi:10.1002/2014JA020275.

[34] Oliveira, D. M., and J. Raeder (2014), Role of interplanetary shock impact angles in substorm triggering, in $P$-MON06, $12^{\text {th }}$ International Conference on Substorms, Ise, Japan.

[35] Oliveira, D. M., and J. Raeder (2015), Impact angle control of interplanetary shock geoeffectiveness: A statistical study, J. Geophys. Res., 120(6), 4313-4323, doi: 10.1002/2015JA021147.

[36] Oliveira, D. M., J. Raeder, B. T. Tsurutani, and J. W. Gjerloev (2015), Geomagnetic activity triggered by interplanetary shocks: The shock impact angle as a controlling factor, in SM41B-2232, AGU Fall Meeting, San Francisco, CA.

[37] Papadopoulos, K. (1979), The role of microturbulence on collisionless reconnection, in Dynamics of the Magnetosphere, edited by S.-I. Akasofu, Astrophysics and Space Science Library 78, pp. 289-309, Springer Netherlands, Amsterdam, The Netherlands, doi:10.1007/ 978-94-009-9519-214.

[38] Pizzo, V. J. (1991), The evolution of corotating stream fronts near the ecliptic plane in the inner solar system: 2 . Three-dimensional tilted-dipole fronts, J. Geophys. Res., 96(A4), 5405-5420, doi:10.1029/91JA00155.

[39] Raeder, J. (2003), Global Magnetohydrodynamics: A Tutorial Review, in Space Plasma Simulation, edited by J. Buchner, C. T. Dum, and M. Scholer, pp. 120, Springer Verlag, Berlin Heilderberg, New York, doi: 10.1007/3-540-36530-311.

[40] Richter, A. K., K. C. Hsieh, A. H. Luttrell, E. Marsch, and R. Schwenn (1985), Review of interplanetary shock phenomena near and within $1 \mathrm{AU}$, in Collisionless Shocks in the Heliosphere: Reviews of Current Research, edited by B. T. Tsurutani and R. G. Stone, Geophys. Monogr. Ser. 35, pp. 33-50, American Geophysical Union, Washington, D.C., doi:10.1029/GM035p0033.

[41] Samsonov, A. A. (2006), Numerical MHD modeling of the Earth's magnetosheath for different IMForientations, Adv. Space Res., 36, 1652-1656, doi:10.1016/j.bbr.2011. 03.031.

[42] Samsonov, A. A. (2011), Propagation of inclined interplanetary shock through the magnetosheath, J. Atmos. Sol. Terr. Phys., 73, 1-9, doi:10.1016/j.bbr.2011.03.031. 
[43] Samsonov, A. A., D. G. Sibeck, and J. Imber (2007), MHD simulation for the interaction of an interplanetary shock with the Earth's magnetosphere, J. Geophys. Res., 112(A12220), 1-9, doi:10.1029/2007JA012627.

[44] Samsonov, A. A., V. A. Sergeev, M. M. Kuznetsova, and D. G. Sibeck (2015), Asymmetric magnetospheric compressions and expansions in response to impact of inclined interplanetary shock, Geophys. Res. Lett., 42(12), 4716-4722, doi:10.1002/2015GL064294.

[45] Schieldge, J., and G. Siscoe (1970), A correlation of the occurrence of simultaneous sudden magnetospheric compressions and geomagnetic bay onsets with selected geophysical indices, J. Atmos. Sol. Terr. Phys., 32(11), 1819-1830, doi:10.1016/0021-9169(70)90139-X.

[46] Schrijver, C. J., R. Dobbins, W. Murtagh, and S. M. Petrinec (2014), Assessing the impact of space weather on the electric power grid based on insurance claims for industrial electrical equipment, Space Weather, doi:10. 1002/2014SW001066.

[47] Siscoe, G. L. (1976), Three-dimensional aspects of interplanetary shock waves, J. Geophys. Res., 81(34), 62356241, doi:10.1029/JA081i034p06235.

[48] Smith, E. J., J. A. Slavin, R. D. Zwickl, and S. J. Bame (1986), Shocks and storm sudden commencements, in Solar Wind and Magnetosphere Coupling, edited by Y. Kamide and J. A. Slavin, p. 345, Terra Scientific, Tokyo, Japan.

[49] Takeuchi, T., C. T. Russell, and T. Araki (2002), Effect of the orientation of interplanetary shock on the geomagnetic sudden commencement, J. Geophys. Res., 107(A12), 1423, doi:10.1029/2002JA009597.

[50] Tsurutani, B., G. Lakhina, O. Verkhoglyadova, W. Gonzalez, E. Echer, and F. Guarnieri (2011), A review of interplanetary discontinuities and their geomagnetic effects, J. Atmos. Sol. Terr. Phys., 73(1), 5-19, doi: 10.1016/j.jastp.2010.04.001.

[51] Tsurutani, B. T., and X. Y. Zhou (2003), Interpla- netary shock triggering of substorms: Wind and Polar, Adv. Space Res., 31(4), 1063-1067, doi:10.1016/ S0273-1177(02)00796-2.

[52] Wang, C., Z. H. Huang, Y. Q. Hu, and X. C. Guo (2005), $3 \mathrm{D}$ global simulation of the interaction of interplanetary shocks with the magnetosphere, in 4th Annual IGPP International Astrophysics Conference on the Physics of Collisionless Shocks, edited by G. Li, G. Zank, and C. T. Russell, pp. 320-324, AIP Conference Proceedings, Am. Inst. of Phys., Washington, D.C., doi:10.1063/1.2032716.

[53] Wang, C., C. X. Li, Z. H. Huang, and J. D. Richardson (2006), Effect of interplanetary shock strengths and orientations on storm sudden commencement rise times, Geophys. Res. Lett., 33(L14104), 1-3, doi:10.1029/ 2006GL025966.

[54] Yue, C., Q. G. Zong, H. Zhang, Y. F. Wang, C. J. Yuan, Z. Y. Pu, S. Y. Fu, A. T. Y. Lui, B. Yang, and C. R. Wang (2010), Geomagnetic activity triggered by interplanetary shocks, J. Geophys. Res., 115(A00I05), 1-13, doi:10.1029/2010JA015356.

[55] Zhou, X., and B. T. Tsurutani (2001), Interplanetary shock triggering of nightside geomagnetic activity: Substorms, pseudobreakups, and quiescent events, J. Geophys. Res., 106(A9), 18,957-18,967, doi:10.1029/ 2000JA003028.

[56] Zhou, X.-Y., and B. T. Tsurutani (1999), Rapid intensification and propagation of the dayside aurora: Large scale interplanetary pressure pulses (fast shocks), Geophys. Res. Lett., 26(8), 1097-1100, doi:10.1029/ 1999GL900173.

[57] Zong, Q.-G., X.-Z. Zhou, Y. F. Wang, X. Li, P. Song, D. N. Baker, T. A. Fritz, P. W. Daly, M. Dunlop, and A. Pedersen (2009), Energetic electron response to ULF waves induced by interplanetary shocks in the outer radiation belt, J. Geophys. Res., 114(A10204), 1-13, doi: 10.1029/2009JA014393. 